\title{
Angst vor Hypoglykämien kann man Patienten und Eltern nehmen
}

\author{
Psychische Probleme verhindern bei manchen Diabetikern eine gute \\ Blutzuckereinstellung. Aufklärung, gegebenenfalls eine Verhaltens- \\ therapie sowie die kontinuierliche Blutzuckermessung oder sensorun- \\ terstützte Pumpentherapie können die Situation verbessern.
}

Der Therapieerfolg bei Diabetes hängt auch von der psychischen Verfassung eines Patienten ab. Angststörungen oder Depressionen kämen bei Diabetespatienten gehäuft vor, so Prof. Karin Lange, Hannover. Die Angst vor Hypoglykämien veranlasse Patienten und Eltern diabeteskranker Kinder zur tendenziell hyperglykämischen Einstellung. Dies führe zu teils chaotischen BZ-Profilen, die mit kontinuierlicher Glukosemessung (CGM) dargestellt werden können.

Das realistische Risiko schwerer Hypoglykämien ist klein und konzentriert sich weitgehend auf Risikogruppen: In einer dänisch-britischen Studie bei 1076 Typ1-Diabetikern traten $54 \%$ aller schweren Hypoglykämien bei nur 5\% der Patienten auf. Die Rate lag bei 0,35 Episoden/ Patientenjahr (Diabetes/Metab Res Rev 2004;20(6):479-86). Hypoglykämiebedingte Tode diabetischer Kinder sind nach Langes Angaben mit einer Häufigkeit von $0,1 / 100.000$ viel seltener als Tode im Straßenverkehr.

Als Ursachen der Ängste nannte die Psychologin unzureichende Kenntnisse und Therapiekompetenz, eine geringe Selbstwirksamkeit und das Verfolgen unrealistischer Therapieziele. Lange riet zur gezielten, individuellen Beratung mit Unterstützung der Selbstwirksamkeit. Hohe Leistungsansprüche müssten hinterfragt und Sicherheit vermittelt werden. Hilfreich dabei sei zudem die CGM mit entsprechender Schulung.

Dass moderne Technik hilft, betonte auch die Lübecker Diabetologin Dr. Simone von Sengbusch: „Durch die sensorunterstützte Pumpentherapie (SUP) zeigen Patienten ein größeres Vertrauen in ihr Diabetesmanagement." So könne das Blutzuckermessgerät Contour ${ }^{\circledast}$ Link die Werte automatisch per Funk an die Medtronic MiniMed ${ }^{\circledR}$ VEO-Pumpe senden, eine manuelle Eingabe erübrige sich. Der Bolusmanager hilft bei der Berechnung der Insulinmenge. Von Sengbusch zitierte eine Studie mit Kindern und jungen Erwachsenen mit Typ-1-Diabetes und mit CGM/SUP. Demnach schätzten $88 \%$ von ihnen vor allem, dass sich Hypoglykämien vermeiden ließen, 83\% gaben eine Abnahme der Hypoglykämieangst an (Pediatric Diabetes 2010; 11:182-7).

Dr. Thomas Meißner

Quelle: Symposium beim Diabeteskongress, 8.5.2013 in Leipzig, Veranstalter: Bayer Vital

\section{Neue Dimension durch Inkretin- therapien erreicht}

\begin{abstract}
Den $\mathrm{HbA}_{1 c}$-Wert effektiv zu senken und trotzdem Hypoglykämien und eine Therapie-induzierte Gewichtszunahme zu vermeiden, ist heute das Ziel der Diabetestherapie. Besonders gut lässt es sich durch GLP-1-basierte Therapien wie DPP-4-Hemmer erreichen.
\end{abstract}

Die Limitationen vieler etablierter Antidiabetika sind groß, wie Prof. Wolfgang E. Schmidt aus Bochum. Vorn steht das Risiko für Hypoglykämien, das lange vernachlässigt wurde. Schwere Hypoglykämien sind unangenehm und steigern die Letalität, ebenso wie der Anstieg des Körpergewichts. Beide Nachteile betreffen v.a. Insulin und Sulfonylharnstoffe.

„Wir haben aus großen Studien gelernt, dass eine glykämische Kontrolle um jeden Preis für jeden kein gutes Prinzip ist", so Schmidt. Eine stringente Senkung des Blutzuckers bringt vor allem Patienten, deren Diabetes noch nicht lange besteht, einen kardiovaskulären Überlebensvorteil. Je länger der Diabetes dauert, desto höher steigen bei intensiver Therapie das Hypoglykämierisiko und die Mortalität.

Mit inkretinbasierten Therapien kommt es weder zu Hypoglykämien noch zu einem Gewichtsanstieg. Denn GLP-1 stimuliert die Insulinfreisetzung glukoseabhängig, indem es die Betazellen empfindlicher gegen Glukose macht. Ist der Glukosespiegel normal, findet keine Stimulation mehr statt. Auch Glukagon wird durch GLP-1 glukoseabhängig runterreguliert. „Diese Eigenschaftenkombination ist phänomenal", so Schmidt.

DPP-4-Hemmer inhibieren den Abbau von GLP-1, sodass dieses länger im Organismus aktiv sein kann. Als erster Vertreter dieser Klasse wurde vor fünf Jahren Sitagliptin (etwa als Xelevia im Handel) verfügbar, das auch das umfassendste Zulassungsspektrum für Monotherapie, Zweier- und Dreierkombinationen hat. Sitagliptin ist auch für Patienten mit Niereninsuffizienz zugelassen und hat in dieser Indikation eine sehr gute Datenlage.

Am häufigsten kommt die Kombination aus Sitagliptin und Metformin (etwa Velmetia ${ }^{\circledR}$ ) zum Einsatz, die ebenfalls seit fünf Jahren verfügbar ist. In einer einjährigen klinischen Studie nahm das Körpergewicht der Patienten mit dieser Medikation ab, in der Kontrollgruppe mit Metformin und Glipizid stieg es - bei ähnlicher $\mathrm{HbA}_{1 \mathrm{c}}$-Senkung. Die Hypoglykämierate lag mit 5\% auf Placeboniveau, in der Kontrollgruppe betrug sie 32\%.

$$
\text { Dr. med. Angelika Bischoff }
$$

Pressekonferenz beim Diabeteskongress am 8.5.2013 in Leipzig, Veranstalter Berlin-Chemie 